\title{
DIFFERENT APPLICATIONS OF PROGRAMMABLE LOGIC CONTROLLER (PLC)
}

\author{
By, Maha M. Lashin \\ Mechanical Engineering Department, Shoubra Faculty of Engineering, Banha University, \\ Egypt.
}

\begin{abstract}
Early Programming Logic Control (PLC) were designed to replace relay logic systems. These PLCs were programmed in "Ladder Logic", which strongly resembles a schematic diagram of relay logic. Programming logic control has several features like protection from the open area conditions such dust, heat and cold. PLC also has the ability to arrangement the inputs/outputs. It has low cost compared with microcontroller systems because using PLC in different applications only required to change the software for each application but in case of using microcontroller the hardware components itself must be changed with different applications.
\end{abstract}

Two important applications for programming logic control and also an engineering solution to save the human life are explained in this paper, one application is a robot used as a toxic chemical substances spraying, and the other application is a robot used for washing the faces glasses of skyscrapers. These mobile robots used PLC as a controlled tool for its motion and liquid flow rate also.

\section{KEYWORDS:}

PLC-Features- Application of PLCs- mobile robot.

\section{INTRODUCTION}

PLC is a special form of microprocessor- based controller. It includes a programmable memory to store instructions and to implement functions such as logic, sequencing, timing, counting and arithmetic as shown in figure1. In order to control machines and processes, PLC is designed to be operated by engineers even by a limited knowledge of computers and computing languages [1]. It has a great advantage of changing the PLC Ladder Diagram after it had been built or worked, which gives the facility of using the same PLC unit for controlling different systems after erasing it each time.

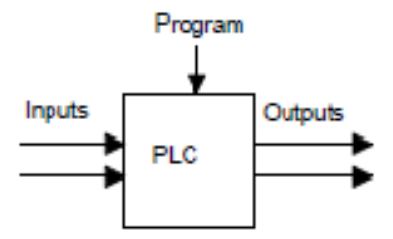

Fig.1: Programmable Logic Controller

DOI : $10.5121 /$ ijcseit.2014.4103 
PLCs could be considered as special computers for industrial use. It does not affected by vibrations, temperature, humidity and noise which mean that it is an open area controller. It could be easily programmed by using a simple programming language which is primarily concerned with logic and switching operations. PLCs contain a hardware and software systems which used for single Input/Output or multi Inputs/Outputs modular systems. PLCs are also used for analog or digital controller systems.

\section{PROGRAMMING LOGIC CONTROL STRUCTURE}

The structure of PLC consisted of two systems, hardware and software systems.

\subsection{PLCs Hardware}

Typically a PLC system has the basic functional components of processor unit, memory, power supply unit, input/output interface section, communications interface and the programming device as explained in [6]. Figure 2 shows the basic arrangement of the PLC hardware components. Also the main task of each component can descriptively as shown in [6] following;

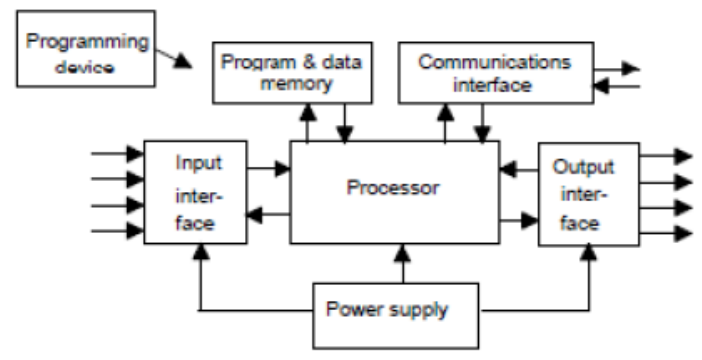

Fig.2: The PLC System

1. Stored the program to interpret the input signals and communicate between this inputs and the output decisions to carries out the control actions done in central processing unit (CPU).

2. The power supply convert AC voltage to DC voltage necessary for the processor and the circuits in the input and output interface modules.

3. The program enters into the memory of the processor by using the programming device and then transferred to the memory unit of the PLC.

4. The program is stored in the memory unit to be used for the control actions to exercised by the microprocessor and data stored from the input for processing and for the output for outputting.

5. The input and output sections are where the processor receives information from external devices and communicates information to external devices.

6. finally the communications interface is used to receive and transmit data by means of communication networks from or to other remote PLCs.

\section{PROGRAMMING PLCs}

Programming devices as in [3] can be a hand-held device, a desktop console or a computer. The program has been designed on the programming device and then transferred to the memory unit of the PLC.

I. Hand-held programming devices will normally contain enough memory to allow the unit to retain programs.

II. Desktop consoles are likely to have a visual display unit with a full keyboard and screen display. 
III. Personal computers are widely configured as program development work-stations.

\section{PLC SOFTWARE PREPARATION}

As explain in [5] the PLC program is executed as a part of repetitive process referred to as a scan. PLC scan starts with the CPU reading the status of inputs. Then the application program is executed using the status of the inputs. Finally when the program is completed, the CPU performs internal diagnostics and communication tasks. Scan cycle ends by updating the outputs, and then starts over. Scan cycle time depends on the size of the program, the number of Inputs/Outputs, and the amount of communication required. Also the control program is built of things called instructions.The instructions are little computer codes that make the inputs and outputs do what the programming want to get the result's need. There are many types of PLC program such as Function Block Diagram (FBD), Statement List (STL), and Ladder Logic Diagram (LAD). LAD is the most famous PLC programming language used. Ladder logic diagram uses components like elements used in a line diagram format to describe hard-wired control. The left vertical line of a LAD represents the power conductor. The output element represents the return path of the circuit.

\section{AREA OF PLC's APPLICATIONS}

PLC represents such a universal controller. It can be used as shown in [1] for different applications and via the program installed in its memory, provides the user with a simple means of changing, extending and optimizing control processes. The original PLC's tasks are involving the interconnection of input signals according to a specified program and if "true" switch the corresponding output. Boolean algebra as explain in [2] forms the mathematical basis for this operation, which recognizes precisely two defined statuses of one variable "0"and "1" and so the output can only assume these two statuses. Timer and counter functions, memory setting and resetting, mathematical computing operations all represent functions, which can be executed by practically any of PLCs. Programming Logic Control using as explained [4] for industrial fields like automatic machines, cars, and robots.

The applications of PLC discussed here are the toxic chemical substances spraying robot and the other is glass-washing robot. PLC used to control the movement of robots, and the flow rate (quantity and time) of different liquids which are used in this robot.

\section{TOXIC CHEMICAL SUBSTANCES SPRAYING ROBOT}

This robot presents solution to the save human health hazards as a result of spraying potentially toxic chemicals in the agriculture field. This is a mobile robot with four wheels to withstand the factors in the farmland or any other places. The robot consists of four systems as shown in Figure 3.

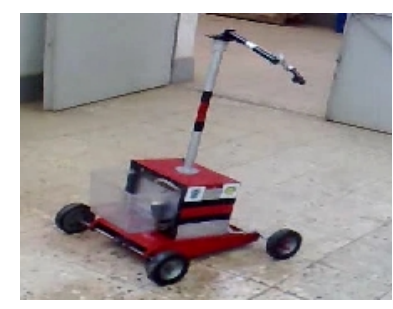

Fig.3: Spraying Robot 
The first is the responsible of the rear wheel drive called the driving system like the drive in car; it forms the gearing system, geared motor and the shaft. The second is the steering system used it. The pumping system used to pump the chemical fluid from the tank into the spraying place. It consists of tank, pump, arm mechanism and nozzle that used in the spraying process. The fourth is the control system represented by PLC used to make the program in which the user orders the robot to do the required tasks. The PLC unit which had been used is a DVP14SS11R2 Delta type with 8 inputs/Outputs. When the on/off button is switched on the robot's drive motor starts working and the robot starts to move forward in straight line for three seconds. The second step starts when the orders come from PLC unit to make the motors of robot's wheels steering to the right direction with 45 angle degree in three seconds. At this position the robot stopped moving and began spraying liquid process for five seconds. After this process has finished the robot's wheels goes back to its origin direction with 45 angle degrees in three seconds. The robots repeat the process in the left direction. After this process has finished the robot's wheels goes back to its origin direction with 45 angle degrees in three seconds, so the robot start to move forward again in straight line for three seconds. It is clear that the complete cycle takes 25 seconds as shown in figure 4.

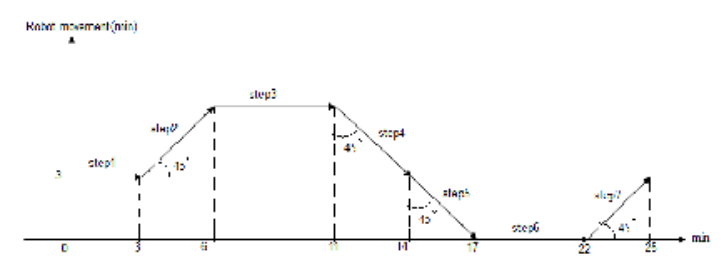

Fig.4: Timing Cycle Diagram of Spray Robot

All the above steps are repeated until the line's end of the agriculture's field. Figure 5 show the Ladder diagram of the PLC unit which represents the movement robot's steps.

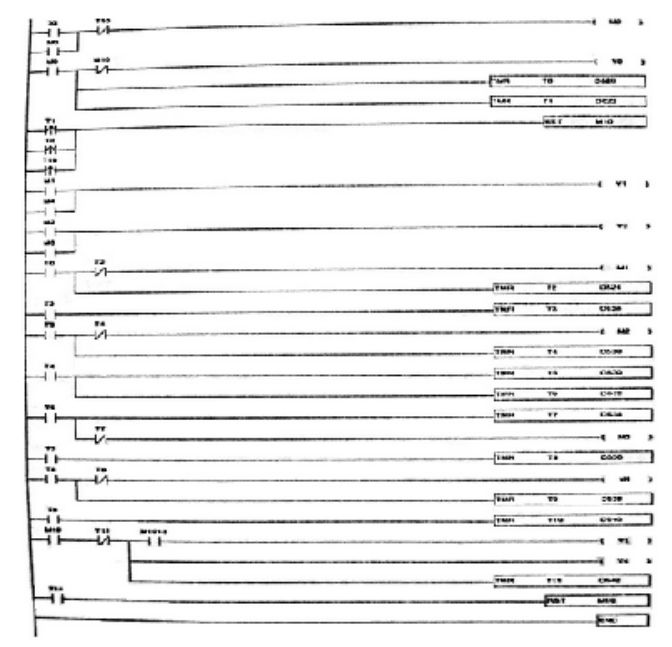

Fig.5: The PLC Ladder Diagram for Spraying Robot 


\section{SKYSCRAPERS'S GLASS WASHING ROBOT}

This robot used for washing the external glass windows of any building. The robot consists of two cars as shown in Figure 6, the first car for the horizontal movement and the second car for vertical movement. The vertical movement car's consists of four motors which are: brushes motor, water pump motor, soap motor and driers motor. The first three motors are working simultaneously and the fourth one has a delay time after them. The car of horizontal motion consists of two motors, one motor for horizontal direction movement, and the other for carrying the car which move in the vertical direction. The horizontal car move at the top edge of the building in forward direction and also carry the vertical car which is responsible for washing process to make it move from up to down for cleaning the windows of the building.
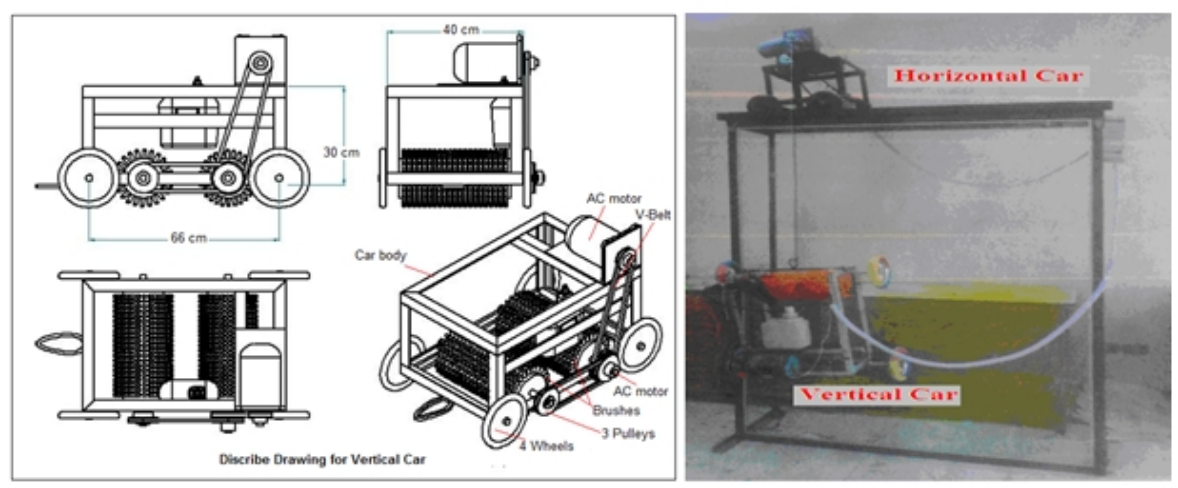

Fig.6: Washing Glass Robot

The robot begins its motion from the roof top of the building by use PLC unit type GE-Fanuc with 9 Input/Outputs. The robot movement begins when the PLC unit's switch turn on then the vertical car starts at the top of the building. The motors of brushes, water pump and drier starts to work. Also the motor which is responsible for moving this car from up to down begins to work. The duration period for vertical motion (from up to down) takes 28 seconds. Followed by five seconds for vertical motion (from down to up). In order to start the second cycle the horizontal car moves horizontal five seconds, so it is clear that the complete cycle takes 38 seconds. All the above steps are repeated until the building face's windows finished. Skyscraper's glass washing robot has been tested on $2 \times 1.5 \mathrm{~m}$ glass window. It takes eight cycles on $4.5 \mathrm{~min}$ to complete cleaning process. Figure 7 show the ladder diagram for the movements of the glass whishing robot. 


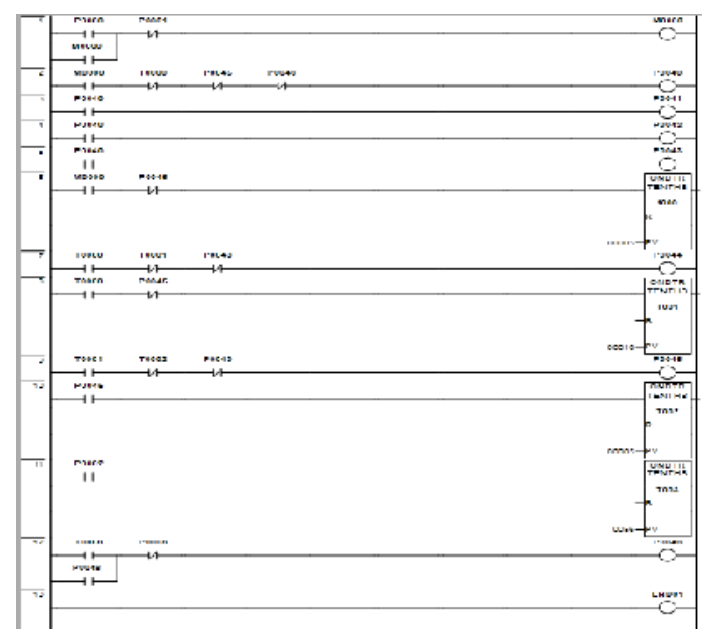

Fig.7: Ladder Diagram of Glass whishing Robot

\section{CONCLUSION}

In this paper the concept of Programmable logic controllers and its application has discussed. PLC applications are typically highly customized systems so the cost of a packaged PLC is low compared to the cost of a specific custom-built controller design. Ladder Diagram programming method used for PLCs units which used in these two applications Two applications had been introduced PLC as a new application of this control system in the spraying's robot and glass washing robot gave a good results. PLC most easier and safe control system for industrial applications like robot.

\section{REFERENCES}

[1] Sanjeev Gupta and S C Sharma "Selection and Application of advance control System: PLC, DCS and PC Based System" Journal of Scientific and Industrial research, April 2005, Vol.64, pp.249-225.

[2] Sadegh Vosough and Amir Vosough "PLC and its Applications" International Journal of Multidisciplinary Sciences and Engineering, November 2011, Vol.2, No.8.

[3] w. Bolton "Programmable Logic Controllers" ELSEVIER, Fourth Edition, 2006.

[4] Hug Jack "Automating Manufacturing Systems with PLCs” April 2005, version4.7.

[5] Gordon Macomb "the Robot Builder's Bonanza" McGraw-Hill professional publishing, Third Edition, 2006.

[6] Jorge Angeles "Fundamentals of Robotic Mechanical Systems : Theory, Methods, and Algorithms" Springer, Third Edition, 2007.

[7] L.A.Bryan and E.A.Bryan "Programmable Controller: Theory and Implementation" An Industrial Text Company Publication, USA, Second edition, 1997.

[8] Prudential PLC-Contact Jackson National Life Insurance Company. Retrieved 18April,2011.

[9] Liptak B G “ Instrumentation Engineer's HandBook: Process Controll” Chilton Book Company I Radnor, PennSylvania, 1999. 